\title{
Oxidation of Electron Donor-Substituted Verdazyls: Building Blocks for Molecular Switches
}

Benjamin C. Haller, ${ }^{\dagger}$ Dallas Chambers, ${ }^{\dagger}$ Ran Cheng, ${ }^{\dagger}$ Victoria Chemistruck, ${ }^{\dagger}$ Timothy F. Hom, ${ }^{\dagger}$ Zhengzheng Li, ${ }^{\dagger}$ Jeffrey Nguyen, ${ }^{\dagger}$ Andrew Ichimura, ${ }^{*}{ }^{\dagger}$ and David J. R. Brook ${ }^{*} \dagger$

${ }^{\dagger}$ Department of Chemistry, San José State University, One Washington Square, San José, California 95192, United States

${ }^{\ddagger}$ Department of Chemistry and Biochemistry, San Francisco State University, 1600 Holloway Avenue, San Francisco, California 94132, United States

\section{Supporting Information}

ABSTRACT: Species that can undergo changes in electronic configuration as a result of an external stimulus such as $\mathrm{pH}$ or solvent polarity can play an important role in sensors, conducting polymers, and molecular switches. One way to achieve such structures is to couple two redox-active fragments, where the redox activity of one of them is strongly dependent upon environment. We report on two new verdazyls, one subsituted with a di-tert-butyl phenol group and the other with a dimethylaminophenyl group, that have the potential for such behavior upon oxidation. Oxidation of both verdazyls with copper(II) triflate in acetonitrile gives diamagnetic verdazylium ions characterized by NMR and UV-
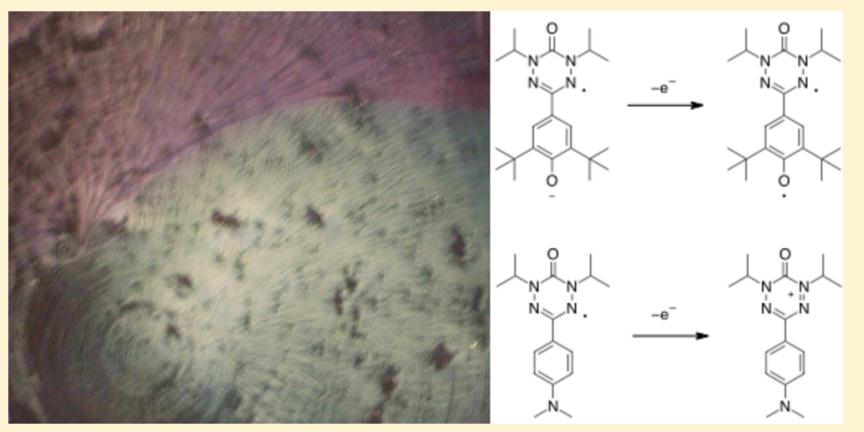
vis spectroscopies. Deprotonation of the phenol-verdazylium results in electron transfer and a switch from a singlet state to a paramagnetic triplet diradical identified by electron spin resonance. The dimethylaminoverdazylium 9 has a diamagnetic ground state, in line with predictions from simple empirical methods and supported by density functional theory calculations. These results indicate that verdazyls may complement nitroxides as spin carriers in the design of organic molecular electronics.

\section{INTRODUCTION}

Electron transfer between organic moieties is particularly interesting as a means of constructing molecular devices, especially when the transfer can be initiated or reversed through an external stimulus. Such stimuli might be chemical, such as changes in $\mathrm{pH}$ or hydrogen bonding, or more subtle such as the application of an electric (or even possibly magnetic) field. The electron transfer itself can be intermolecular (such as the acidinduced disproportionation of verdazyl ${ }^{1,2}$ or nitronyl-nitroxide ${ }^{3}$ radicals), but for application as a switch or other device the two components should be constrained. This is typically via covalent bonds but also possibly through a crystal lattice or other well-defined supramolecular interaction. An important example is the acid doping of polyaniline, in which protons induce the comproportionation of diaminobenzene and quinone diiimine sites within the polymer to generate charge carriers and change the material from an insulator to a conductor. $^{4,5}$

Other recent examples include electron transfer between a dianisylamine and quinone diimine group initiated by acid ${ }^{6}$ and the system $\mathbf{1 a}-\mathbf{1 b}$ reported in 1997 by Ishiguro and co-workers from oxidation of the corresponding phenol ${ }^{7}$ (Scheme 1). The latter case is particularly interesting because the resulting openshell species has a triplet ground state. A related system is the dimethylamino system (2) reported by Sakurai where oxidation preferentially occurs at the dimethylaminophenyl ring to give a triplet diradical. ${ }^{8}$

Though many studies have focused on nitronyl nitroxides, verdazyls are particularly interesting in switching applications because the two substituents at the 1 and 5 positions of the verdazyl ring can be replaced with aryl groups, and oxidation or reduction of the verdazyl may turn on or off magnetic or conductive pathways between these groups. We report on two verdazyls related to the nitronyl nitroxides reported by Sakurai and Ishigura, namely, the di-tert-butyl phenol verdazyl 3 and the dimethylaminophenyl verdazyl 4 . The former has not been previously reported, and while the latter was mentioned in an earlier publication, ${ }^{9}$ we report here on a more detailed investigation of its chemistry and electronic structure. In particular, we seek to determine if the verdazyls 3 and $\mathbf{4}$ are comparable in behavior to the corresponding nitronyl nitroxides, and in so doing, possibly gain some insight as to whether the switching between ground states observed in $\mathbf{1}$ and the oxidation to give a diradical observed in $\mathbf{2}$ can be reproduced in other systems.

Received: September 23, 2015

Published: October 9, 2015 
Scheme 1<smiles>CC(C)(C)c1cc(C2=[N+]([O-])C(C)(C)C(C)(C)[N+]2=O)cc(C(C)(C)C)c1O</smiles>

$1 a$

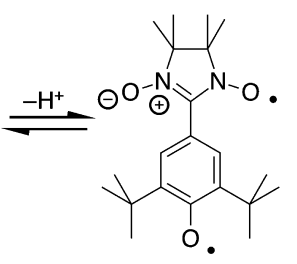

16<smiles></smiles>

2

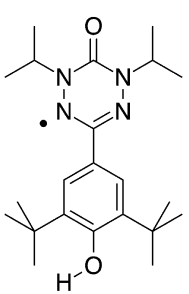

3

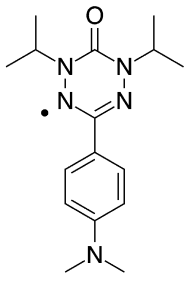

4

Scheme 2. Acid/Base and Redox Chemistry of Radical $3^{a}$<smiles>C=CC#CC(=O)O[SnH3]</smiles><smiles></smiles>

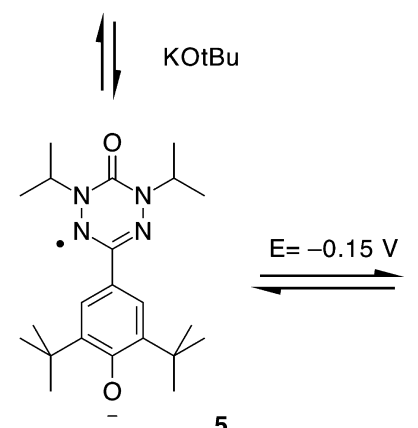

$\mathrm{H}^{+} \| \mathrm{Et}_{3} \mathrm{~N}$
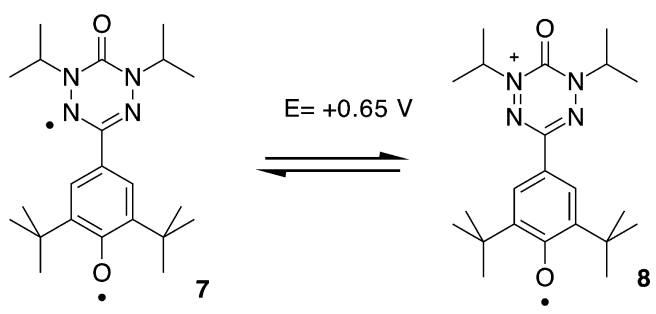

${ }^{a}$ Electrochemical potentials are reported vs standard SCE.

\section{RESULTS}

The 3-(3',5'-di-tert-butyl-4'-hydroxy-phenyl)-verdazyl 3 was synthesized from the corresponding tetrazane (2,4-diisopropyl-6-(3,5-di-tert-butyl-4-hydroxyphenyl)-1,2,4,5-tetrazinan-3one) using previously reported procedures. ${ }^{9,10}$ It crystallizes in dark purple-red blocks from methanol. Radical 3 is typical for 3aryl-6-oxoverdazyls, giving red solutions in organic solvents. The electron spin resonance (ESR) spectrum shows coupling to all four nitrogen atoms in the ring along with the two isopropyl methine hydrogens. The electronic spectrum in acetonitrile shows two maxima in the visible region at $418 \mathrm{~nm}$ $\left(\varepsilon=990 \mathrm{~L} \mathrm{~mol}^{-1} \mathrm{~cm}^{-1}\right)$ and $521 \mathrm{~nm}\left(\varepsilon=640 \mathrm{~L} \mathrm{~mol}^{-1} \mathrm{~cm}^{-1}\right)$. Deprotonation of 3 to give radical-anion 5 (Scheme 2) results in only small changes in the ESR parameters but a large, solvent-dependent red shift of the low-energy band.

In methanol the low-energy band of 5 has a maximum at 665 $\mathrm{nm}$, whereas in acetonitrile the band maximum is at $785 \mathrm{~nm}$. Spectra of the radical 3 in methanol and radical-anion 5 in methanol and acetonitrile are shown in Figure 1.

Earlier results with verdazyl phenols ${ }^{9}$ established that the long wavelength transition in the electronic spectrum has significant charge transfer (CT) character between the electronrich aryl substituent and the verdazyl singly occupied molecular orbital (SOMO). Deprotonation of verdazyl phenoxides resulted in a hydrogen bond-dependent red shift of the band resulting from the increased electron density of the aryl ring. The spectra for $\mathbf{3}$ and $\mathbf{5}$ are also consistent with this picture. For 3 , the two additional alkyl substituents on the aryl ring result in

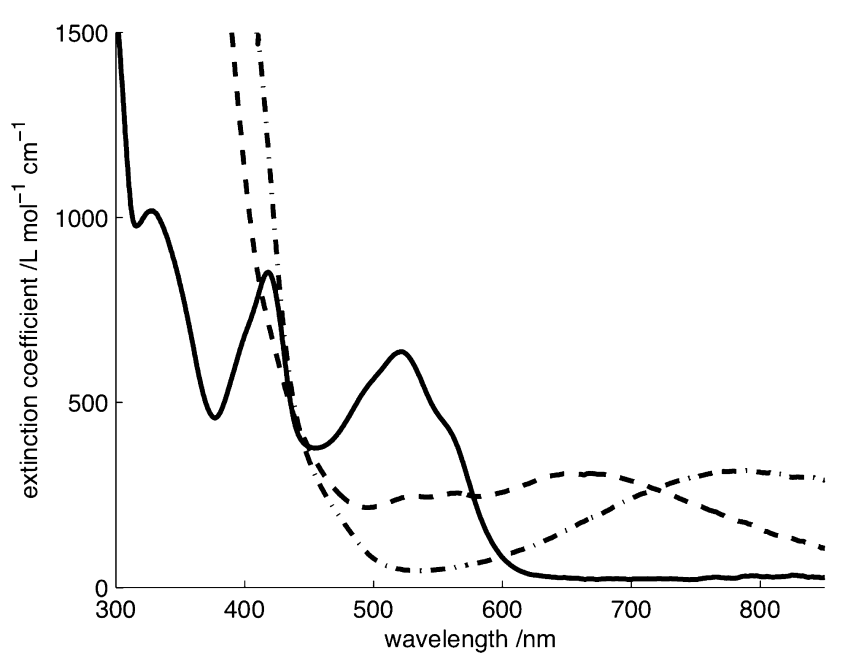

Figure 1. Electronic spectra of radical 3 in methanol (solid line) along with spectra of radical anion $\mathbf{5}$ in methanol $(--)$ and acetonitrile $(-$. $-)$.

a slight red shift of the low-energy electronic transition compared with the unsubstituted verdazyl phenol. ${ }^{9}$ For phenoxide 5, the steric bulk of the tert-butyl subsituents limits hydrogen bonding and results in significantly greater red shifts compared to the previously reported verdazyl phenolate; ${ }^{9}$ even in polar, strong $\mathrm{H}$-bond donor solvents the $\mathrm{CT}$ absorption is above $650 \mathrm{~nm}$, and in non-hydrogen bonding solvents such as 
acetonitrile, the absorbance maximum is near $800 \mathrm{~nm}$ with a tail into the infrared.

Cyclic voltammetry on 3 in acetonitrile revealed a reversible oxidation at $+0.51 \mathrm{~V}$ versus standard calomel electrode (SCE) in the range expected for the verdazyl/verdazylium couple. ${ }^{9,11}$ Upon deprotonation to 5 this oxidation potential shifted to $+0.65 \mathrm{~V}$, and an additional reversible oxidation wave was observed at $-0.15 \mathrm{~V}$ corresponding to the phenoxyl/phenoxide couple. Additional waves corresponding to decomposition of the oxidation products were also observed at slower scan rates.

To further characterize the oxidation products, samples of 3 were oxidized in acetonitrile by addition of copper(II) triflate and monitored by UV-vis, NMR, and ESR. Oxidation in acetonitrile resulted in a color change of the solution from red to deep blue, with a corresponding shift of the longest wavelength absorption to $591 \mathrm{~nm}\left(\varepsilon=510 \mathrm{~L} \mathrm{~mol}^{-1} \mathrm{~cm}^{-1}\right.$; Figure 2). ${ }^{1} \mathrm{H}$ NMR revealed a well-resolved spectrum consistent with the formation of the diamagnetic verdazylium cation 6.

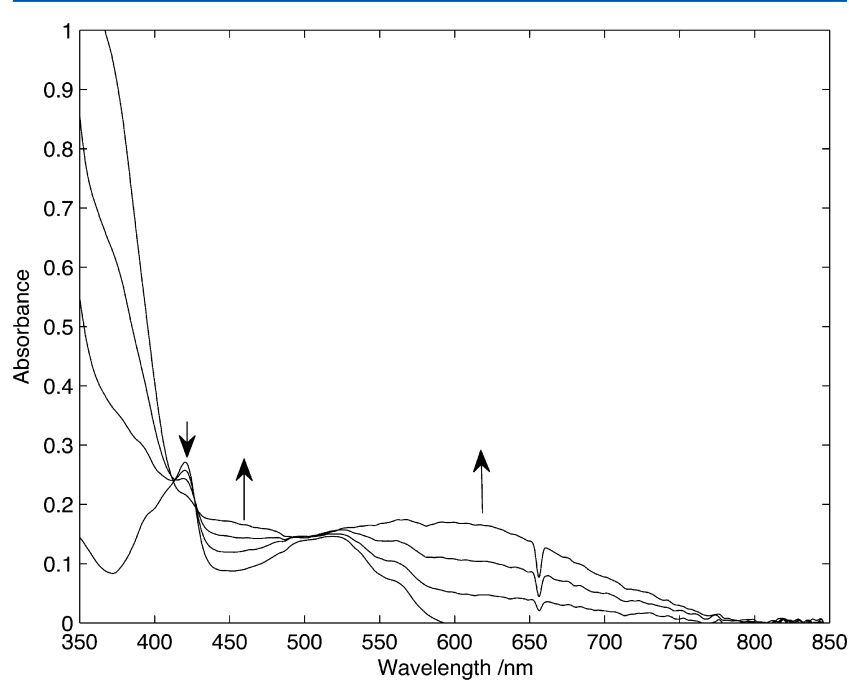

Figure 2. Oxidation of radical 3 to verdazylium 6 with copper triflate in acetonitrile, monitored by UV-vis spectroscopy. Spectra correspond to addition of zero, $0.33,0.66$, and 1 equiv of $\mathrm{Cu}^{2+}$.

The red shift in the UV-vis spectrum is also consistent with the product being a verdazylium cation since oxidation increases the electron affinity of the tetrazane ring, lowering the energy required for CT between the ring systems.

Addition of triethylamine to acetonitrile solutions of 6 results in a second change in color from blue to orange and appearance of new maxima at $422 \mathrm{~nm}\left(\varepsilon=1050 \mathrm{~L} \mathrm{~mol}^{-1} \mathrm{~cm}^{-1}\right)$ and 484 $\mathrm{nm}\left(\varepsilon=1280 \mathrm{~L} \mathrm{~mol}^{-1} \mathrm{~cm}^{-1}\right.$; Figure 3$)$. At the same time the ${ }^{1} \mathrm{H}$ NMR spectrum is completely lost indicating that the deprotonated species has, at the least, a thermally populated paramagnetic state. ESR of frozen solutions gives signals corresponding to a randomly oriented triplet species 7 (Figure 4) with a half-field signal and zero field splitting parameters $|D|$ $=0.0275 \mathrm{~cm}^{-1}$ and $E=0.0025 \mathrm{~cm}^{-1}$. A strong doublet signal at $g=2.004$ is also observed, which may correspond to unoxidized radical or doublet decomposition products of the diradical. Variable-temperature measurements of the ESR signal intensity over the range of 4-100 K shows Curie behavior for the halffield signal (Supporting Information) indicating that the triplet state is the ground state or that the triplet and lowest singlet

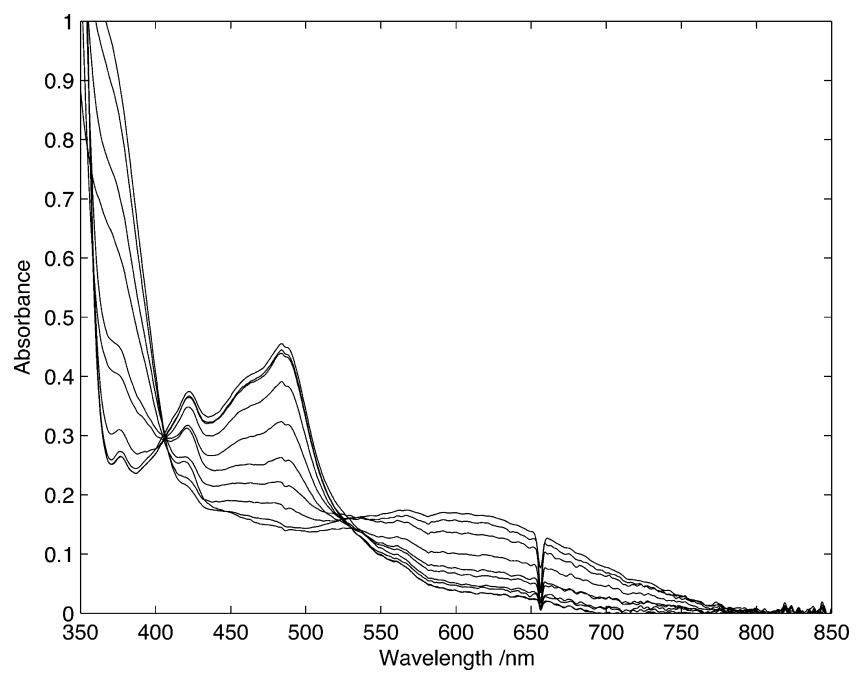

Figure 3. Deprotonation of 6 with triethylamine to give 7 monitored by UV-visible spectroscopy.

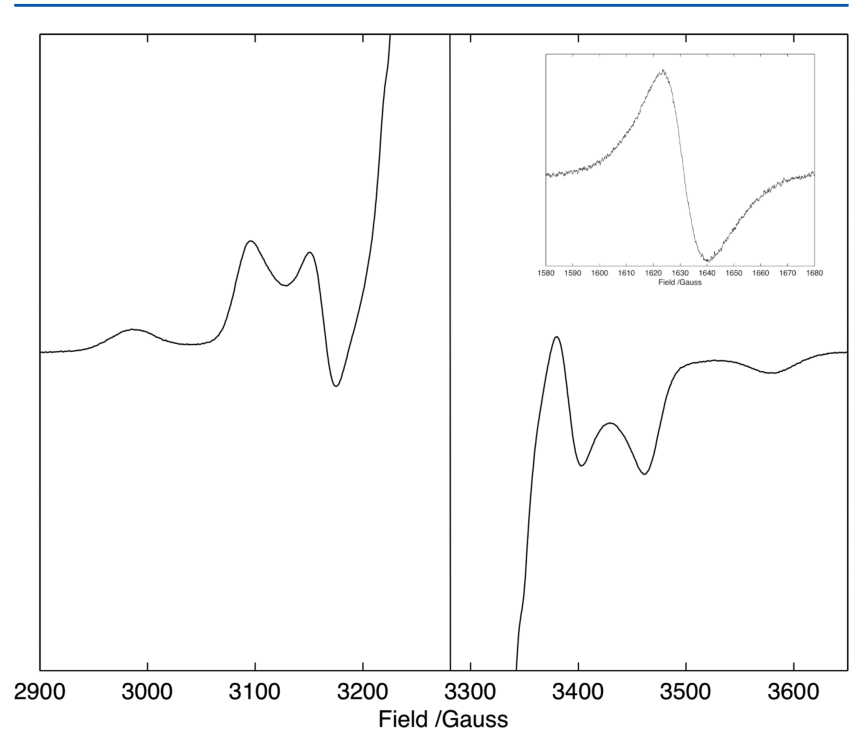

Figure 4. ESR spectrum of triplet diradical 7 at $4 \mathrm{~K}$. (inset) The halffield signal.

state(s) are nearly degenerate in energy. Oxidation and acidbase chemistry of 3 is summarized in Scheme 2.

Diradical 7 was examined with density functional theory (DFT) to clarify our understanding of its electronic structure (see Experimental Section). In these calculations, methyl groups were substituted for isopropyl on the verdazyl and aromatic rings (structure $7^{\prime}$ ) to keep charge donation effects while increasing the computational efficiency. The geometries of the closed-shell singlet and open-shell triplet and singlet states were optimized with the B3LYP hybrid functional and 6$311 \mathrm{~g}^{* *}$ basis set. Broken symmetry (BS) unrestricted DFT wave functions $^{12-15}$ (U-DFT) were used for the $M_{S}=1$ and $M_{S}$ $=0$ (open-shell) states, while the closed-shell $\mathrm{M}_{\mathrm{S}}=0$ wave function employed the restricted formalism (R-DFT). The singlet-triplet $(E(S)-E(T))$ energy difference between openshell states can be reliably predicted with BS DFT wave functions using the B3LYP/6-311g** model chemistry. ${ }^{13,15-22}$ Physically, the BS unrestricted wave functions correspond to the diradical triplet and singlet states with an unpaired electron localized to the verdazyl and phenoxyl rings. The R-DFT model 
Table 1. Relative Energies (hartrees) of the Spin-Restricted $\left(M_{S}=0\right)$ and Broken-Symmetry $\left(M_{S}=0\right.$ and $\left.M_{S}=1\right)$ States of $7^{\prime a}$

\begin{tabular}{|c|c|c|c|c|c|c|c|}
\hline & R-DFT $(M s=0)$ & U-DFT $(\mathrm{Ms}=0)$ & $\mathrm{U}-\mathrm{DFT}(\mathrm{Ms}=1)$ & $\left\langle S^{2}\right\rangle_{\mathrm{M}_{\mathrm{S}}=0}^{\mathrm{R}}$ & $\left\langle S^{2}\right\rangle_{\mathrm{M}_{\mathrm{S}}=0}^{\mathrm{U}}$ & $\left\langle S^{2}\right\rangle_{\mathrm{M}_{\mathrm{S}}=1}^{\mathrm{U}}$ & $\mathrm{SDF}^{b}$ \\
\hline$c$ & $0.031817^{d}$ & 0.020579 & 0.0 & 0.0 & 0.9813 & 2.0554 & \\
\hline $\mathrm{CH}_{3} \mathrm{CN}^{e}$ & 0.023402 & 0.015882 & 0.0 & 0.0 & 0.7985 & 2.0518 & \\
\hline $\mathrm{w} / \mathrm{SDF}^{c, b}$ & & 0.039380 & 0.0 & & & & 1.9136 \\
\hline $\mathrm{CH}_{3} \mathrm{CN} \mathrm{SDF}{ }^{f}$ & & 0.026001 & 0.0 & & & & 1.6371 \\
\hline
\end{tabular}

${ }^{a}$ Expectation values of $\left\langle\mathrm{S}^{2}\right\rangle$ for each state and the SDF are also shown. Calculations employed the B3LYP/6-311g** level of theory. ${ }^{b}$ Spin decontamination factor defined in eq $2 .{ }^{c}$ Gas-phase calculation. ${ }^{d}$ Energies are reported in hartrees relative to the lowest-energy state. ${ }^{e} \mathrm{Geometries}$ were optimized including the effects of solvent, acetonitrile, using the PCM. ${ }^{f}$ Relative energy including the PCM and SDF.

is well-suited for the closed-shell state, which may be described as a verdazylium cation bonded to a phenoxide anion. Since the optical spectra shifted with oxidation state, the effect of solvent on the energy differences was approximated using the polarizable continuum model (PCM) with acetonitrile as the solvent. $^{23}$ The molecular geometries were also optimized at the B3LYP/6-311G** level with the PCM model.

Table 1 shows the relative energies and expectation values of $\hat{S}^{2}$ of the three lowest electronic states of $7^{\prime}$ (absolute energies are reported in the Supporting Information). The triplet state of $7^{\prime}$ was found to be 12.9 and $20.0 \mathrm{kcal} / \mathrm{mol}$ lower in energy than the open-shell and closed-shell singlet states, respectively. When solvent effects are included using the continuum model, the singlet-triplet gap decreases to 10.0 and $14.7 \mathrm{kcal} / \mathrm{mol}$ for the open- and closed-shell singlet states, respectively. A triplet ground state for $7^{\prime}$ for both gas-phase and PCM calculations agrees with the ESR data of 7 in which the intensity of the $\Delta \mathrm{M}_{S}$ $=2$ transition exhibited a $1 / T$ dependence. The energy of the charge-separated R-DFT $(\mathrm{Ms}=0)$ state is lowered more than the U-DFT $(\mathrm{Ms}=0)$ state when solvent is included for two reasons. As expected, the polar solvent helps to stabilize the charge-separated state more than the open-shell state even with the simple PC model. Second, the U-DFT $\left(M_{S}=0\right)$ wave function has $\left\langle\hat{\mathrm{S}}^{2}\right\rangle$ equal to 0.7985 , which is low compared to the expected value of $\sim 1$ as observed for the gas-phase calculation. The BS singlet wave function in its simplest two electron-two orbital form can be thought of as an even mixture of $\mathrm{M}_{\mathrm{S}}=0$ singlet and triplet states, $\Phi(\mathrm{BS})=2^{-1 / 2}[\Phi(0,0)+\Phi(1,0)]$, where $\Phi(\mathrm{S}, \mathrm{Ms})=\Phi(0,0)=2^{-1 / 2}[|a b \alpha \beta|-|a b \beta \alpha|]$ (singlet) and $\Phi(1,0)=2^{-1 / 2}[|a b \alpha \beta|+|a b \beta \alpha|]$ (triplet), $a$ and $b$ are orbitals, and $\alpha$ and $\beta$ are opposite spins. ${ }^{13}$ In the gas phase, the U-DFT $\mathrm{M}_{\mathrm{S}}=0$ state has diradical character consistent with $\left\langle\hat{\mathrm{S}}^{2}\right\rangle$ $=0.98 .^{18,22}$ However, the closed-shell components have greater weight in the PCM calculation due to stabilization of the ionic terms by the continuum electric field, which is shown by the lower value of $\left\langle\hat{\mathrm{S}}^{2}\right\rangle .^{24,25}$

So far, we have assumed that the open-shell singlet-triplet energy gap is given by $E(\mathrm{BS})-E(\mathrm{~T})$. However, the spatial orbitals in a U-DFT calculation need not be orthogonal. ${ }^{13,18,24,25}$ Furthermore, the core (closed-shell) orbitals may have induced spin polarization, ${ }^{16,25-27}$ which has physical meaning (e.g., spin density at magnetic nuclei) especially for the triplet state $(\Phi(1,1)=|a b \alpha \alpha| ; \Phi(1,-1)=|a b \beta \beta|)$. Malrieu and Trinquier ${ }^{25}$ proposed a more sophisticated expression for the energy difference that corrects for spin contamination as a result of the unrestricted formalism (eq 1 ).

$$
E(\mathrm{~S})-E(\mathrm{~T})=\lambda[E(\mathrm{BS})-E(\mathrm{~T})]
$$

where $\lambda$, the $\mathrm{SDF}^{25}$ eq 2 , is given by

$$
\lambda=\frac{\left\langle S^{2}\right\rangle_{T}}{\left\langle S^{2}\right\rangle_{T}-\left\langle S^{2}\right\rangle_{B S}}
$$

Notice that when $\left\langle\hat{\mathrm{S}}^{2}\right\rangle_{\mathrm{BS}}=0$ as it should for a closed-shell singlet, $\lambda=1$, and the energy difference equals $E(\mathrm{BS})-E(\mathrm{~T})$. At the other extreme, when $\left\langle\hat{S}^{2}\right\rangle_{\mathrm{BS}}=1$, the energy gap is given by $2[E(\mathrm{BS})-E(\mathrm{~T})]$. The advantage of the SDF is that the correct behavior is obtained in the limiting cases and over the range for $0 \leq\left\langle\hat{\mathrm{S}}^{2}\right\rangle_{\mathrm{BS}} \leq 1$.

Table 1 shows that the open-shell singlet is 24.7 and 16.3 $\mathrm{kcal} / \mathrm{mol}$ higher than the triplet state for the gas-phase and PCM models. When spin contamination is corrected, the openshell singlet is 2.6 mhartree higher than the R-DFT prediction. The stabilization of the open-shell singlet due to the solvation model is reproduced when the energy difference is computed with eq 1 .

Structural parameters such as bond lengths and bond angles are very similar for the ground-state triplet and open-shell singlet states of $7^{\prime}$, but both differ somewhat from the closedshell singlet state (Cartesian coordinates and selected bonding parameters are included in the Supporting Information). The most notable changes occur between the $\mathrm{N}-\mathrm{N}$ bonds of the verdazyl group, which are longer by $0.033 \AA$, and the $\mathrm{C}-\mathrm{C}$ bond that connect verdazyl and phenoxyl is $0.008-0.018 \AA$ shorter.

The dimethylaminophenyl verdazyl 4 was synthesized as previously reported. ${ }^{9}$ While initially synthesized as dark green crystals from methanol/water, rapid evaporation of methanol solutions gives mixtures of two different crystal phases, one purple and the other green (see Table of Contents figure). Examination of reflectance spectra of the two forms indicates that the green form has an absorption maximum at $605 \mathrm{~nm}$, while that of the purple form is $580 \mathrm{~nm}$. Reflectance spectra are provided in the Supporting Information.

Further experiments allowed us to grow single crystals of both phases suitable for X-ray diffraction study. Evaporation of heptane solutions gave the purple phase, space group $P 2_{1}$, in which the dimethylamino group is distinctly pyramidal. In this structure the nitrogen is 0.205 (4) $\AA$ from the plane defined by the three carbon atoms it is attached to. Neither $\mathrm{N}-\mathrm{CH}_{3}$ bond is coplanar with the phenyl ring, while the dihedral between the phenyl and verdazyl rings is $22.70(3)^{\circ}$. In the green phase (from methanol/water) the dimethyl amino group is essentially planar-the distance between the nitrogen and the plane defined by the attached carbons is $0.025(4) \AA$. The dimethylamino group is almost coplanar with the phenyl group (the dihedral angle is $4.26(3)^{\circ}$ ), though the dihedral between phenyl and verdazyl rings is slightly larger $\left(27.28(3)^{\circ}\right)$. Inspection of packing diagrams show the dimethylaminophenyl group is $\pi$-stacked with that from a neighboring molecule with the relatively large $\pi$-stacking distance of 3.690 (4) $\AA$. (Figure 5). Crystallographic cell parameters are summarized in Table 2. Full structural data and experimental details are given in the Supporting Information in .cif format. 


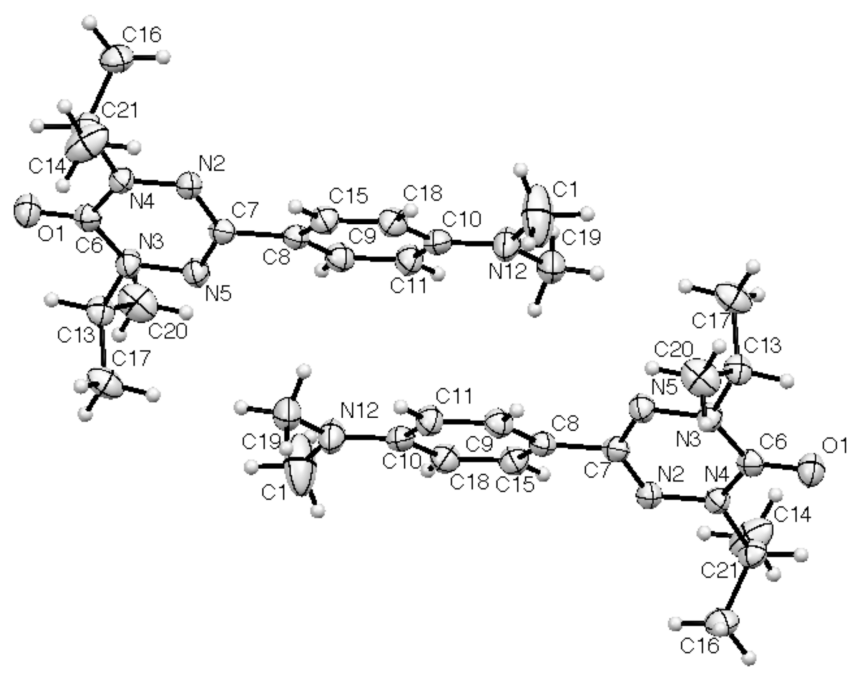

Figure 5. $\pi$-stacked dimer of dimethylamino verdazyl 4 in the green $\left(P 2_{1} / n\right)$ polymorph.

Table 2. Unit Cell Parameters for the Two Forms of Verdazyl 4

$\begin{array}{lll}\text { color } & \text { purple } & \text { green } \\ \text { empirical formula } & \mathrm{C}_{16} \mathrm{H}_{24} \mathrm{~N}_{5} \mathrm{O} & \mathrm{C}_{16} \mathrm{H}_{24} \mathrm{~N}_{5} \mathrm{O} \\ \text { formula weight } & 302.4 & 302.4 \\ \text { temperature, } \mathrm{K} & 100 \mathrm{~K} & 100 \mathrm{~K} \\ \text { crystal system } & \text { monoclinic } & \text { monoclinic } \\ \text { space group } & P 2_{1} & P 2_{1} / n \\ a, \AA & 8.054(3) & 5.889(3) \\ b, \AA & 5.950(2) & 14.574(7) \\ c, \AA & 17.507(6) & 19.326(10) \\ \alpha, \text { deg } & 90.0 & 90.0 \\ \beta, \text { deg } & 95.310(5) & 93.463(8) \\ \gamma, \text { deg } & 90.0 & 90.0 \\ V, \AA \AA^{3} & 835.4(5) & 1655.9(15) \\ \mathrm{Z} & 2.0 & 4.0 \\ \rho_{\text {calc }} \mathrm{mg}, \mathrm{mm}^{3} & 1.2021 & 1.2129\end{array}$

The crystallographic data suggest an explanation for the differences in color, consistent with the intramolecular CT nature of the long wavelength transition. Specifically, the neighboring $\pi$-stacked ring increases the electron density at the dimethylaminophenyl group and lowers its ionization potential. A similar effect was observed in dimethylamino-substituted $[3,3]$ paracyclophanes. ${ }^{28}$ This in turn reduces the energy of the CT transition and results in the color change.

Despite the differences in molecular interaction, magnetic susceptibility measurements on both forms of $\mathbf{4}$ show essentially Curie behavior down to $5 \mathrm{~K}$, indicating that there is very little interaction between unpaired electrons on neighboring molecules. Plots of magnetic susceptibility data are provided in the Supporting Information.

Electrochemistry of $\mathbf{4}$ in acetonitrile has been previously reported $^{9}$ and shows reversible oxidation waves at $+0.54 \mathrm{~V}$ and $+1.1 \mathrm{~V}$ versus SCE. Oxidation of 4 with 1 equiv of $\mathrm{Cu}(\mathrm{OTf})_{2}$ in acetonitrile resulted in a color change of the solution from purple to green. UV-vis spectroscopy indicates that the longest wavelength absorption of this solution tails into the near-IR with a maximum near $800 \mathrm{~nm}$ (Figure 6). ${ }^{1} \mathrm{H}$ NMR of the solution gave a spectrum consistent with a verdazylium cation 9 (Scheme 3); however, unlike the phenol species there was

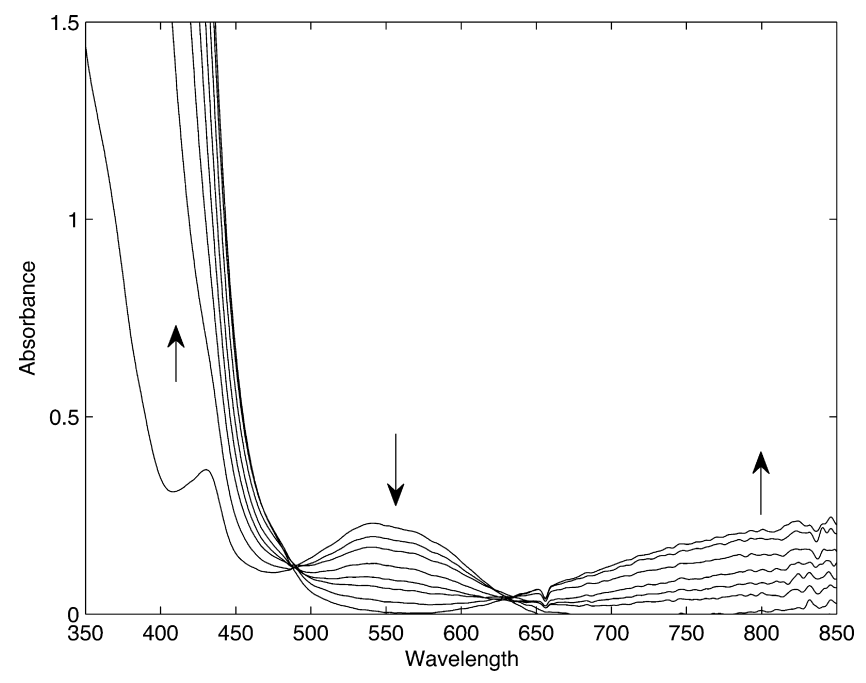

Figure 6. Oxidation of radical 4 monitored by UV-vis.

Scheme 3. Acid/Base and Redox Chemistry of Radical $4^{a}$

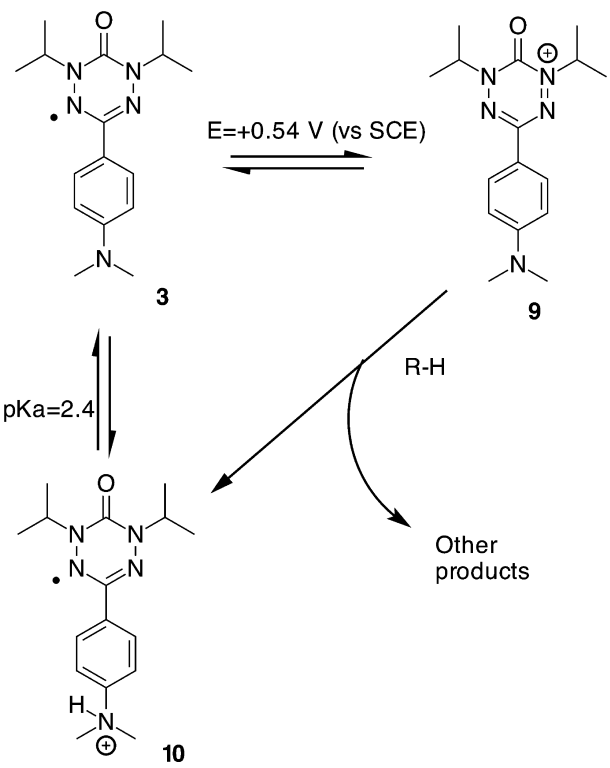

${ }^{a_{T}}$ The $\mathrm{p} K_{\mathrm{a}}$ of species 10 was reported in an earlier publication by Chemistruck et al. (ref 9).

some broadening of the aromatic resonances, along with what appears to be another species with an aromatic ring. At liquid nitrogen temperature, there was no evidence for the triplet state in frozen solutions of 9 by ESR spectroscopy.

When allowed to stand at room temperature for ca. $10 \mathrm{~min}$, (either in air or under $\mathrm{N}_{2}$ ) solutions of verdazylium 9 decomposed to give orange solutions. The decomposition was noticeably faster when oxidation was incomplete; under these conditions the major product was the protonated form of the free radical 10. The identity of this product was confirmed by X-ray crystallography and by comparison of the UV-vis spectrum with authentic samples (Full details of crystallographic data collection and structure solution are provided in the Supporting Information).

As with diradical 7, computational studies on the methylsubstituted analogue $\mathbf{9}^{\prime}$ were used to further understand this system, Table 3. The lowest-energy state of cation $\mathbf{9}^{\prime}$ is the UDFT $\left(M_{S}=0\right)$ singlet state. The average value of the $\hat{S}^{2}$ 
Table 3. Relative Energies (hartrees) of the Spin-Restricted $(\mathrm{Ms}=0)$ and Broken-Symmetry $(\mathrm{Ms}=\mathbf{0}$ and $\mathrm{Ms}=1)$ States of $9^{\prime}{ }^{a}$

\begin{tabular}{|c|c|c|c|c|c|c|c|}
\hline & R-DFT $(\mathrm{Ms}=0)$ & $\mathrm{U}-\mathrm{DFT}(\mathrm{Ms}=0)$ & U-DFT $(\mathrm{Ms}=1)$ & $\left\langle S^{2}\right\rangle_{\mathrm{M}_{\mathrm{S}}=0}^{\mathrm{R}}$ & $\left\langle\mathrm{S}^{2}\right\rangle_{\mathrm{M}_{\mathrm{S}}=0}^{\mathrm{U}}$ & $\left\langle S^{2}\right\rangle_{M_{S}=1}^{\mathrm{U}}$ & $\mathrm{SDF}^{b}$ \\
\hline$c$ & $0.002522^{d}$ & 0.0 & 0.003727 & 0.0 & 0.4463 & 2.0364 & \\
\hline $\mathrm{CH}_{3} \mathrm{CN}^{e}$ & 0.000157 & 0.0 & 0.002328 & 0.0 & 0.1500 & 2.0463 & \\
\hline $\mathrm{w} / \mathrm{SDF}^{c, b}$ & & 0.0 & 0.004773 & & & & 1.2807 \\
\hline $\mathrm{CH}_{3} \mathrm{CN} \mathrm{SDF}{ }^{f}$ & & 0.0 & 0.002513 & & & & 1.0791 \\
\hline
\end{tabular}

${ }^{a}$ Expectation values of $S^{2}$ for each state and the SDF are also shown. Calculations employed the B3LYP/6-311g** level of theory. ${ }^{b}$ SDF defined in eq 2. ${ }^{c}$ Gas-phase calculation. ${ }^{d}$ Energies are reported in hartrees relative to the lowest energy state. ${ }^{e}$ Geometries were optimized including the effects of solvent, acetonitrile, using the PCM. ${ }^{f}$ Relative energy including the PCM model and SDF defined in eq 2.

operator for the BS singlet state equals 0.45 and 0.15 for the gas-phase and PCM models, respectively. These $\hat{S}^{2}$ values are significantly less than 1 and indicate the large weight of the closed-shell component in the BS wave function. For the singlet $\mathrm{Ms}=0$ state calculated with solvation, the R-DFT and U-DFT results are essentially identical differing by only $0.1 \mathrm{kcal} / \mathrm{mol}$, which supports the closed-shell interpretation. In the gas phase, the R-DFT singlet is $1.6 \mathrm{kcal} / \mathrm{mol}$ higher than the BS-DFT. The triplet state is a low-lying excited state $1.5 \mathrm{kcal} / \mathrm{mol}$ higher than the BS-DFT result including the effect of solvent. The SDF has a small effect on the energy splitting due to the largely closed-shell character of $\mathbf{9}^{\prime}$. The gas-phase energy difference calculated with eq 1 places the triplet state $3.0 \mathrm{kcal} / \mathrm{mol}$ higher than the lowest singlet. The best description of the bonding in this cation is given by resonance structure 9. Bond lengths between verdazylium nitrogen atoms are shorter in the R-DFT singlet compared to the U-DFT triplet, and the $\mathrm{C}-\mathrm{N}$ bond of the dimethylamino group of the singlet state is longer than the triplet, which suggests localization of the positive charge to the verdazylium group. The U-DFT and R-DFT singlet geometries are virtually identical for 9', especially with the PCM model, just as the U-DFT triplet and singlet geometries are similar for $7^{\prime}$ (Cartesian coordinates and selected bonding parameters are included in the Supporting Information).

The DFT results for $7^{\prime}$ and $9^{\prime}$ can be summarized as follows: (1) The energy differences predicted by a combination of BSDFT and R-DFT, with and without the SDF and over a range of BS-DFT Ms $=0\left\langle\hat{S}^{2}\right\rangle$ values, are consistent with experiment in which a ground-state triplet was found for 7 and a singlet for 9. This is true for both "gas-phase" calculations and under the influence of acetonitrile using the PCM model. (2) The singlet structures predicted by BS-DFT are consistent with the open and closed-shell nature of 7 and 9, respectively; the open-shell U-DFT triplet and singlet states of $7^{\prime}$ have similar geometries, and those of the R-DFT and BS(U)-DFT singlet states of $\mathbf{9}^{\prime}$ are also very similar.

The chemistry of verdazyl 4 is summarized in Scheme 3.

\section{DISCUSSION}

While DFT calculations can provide considerable insight into structure and energetics of various electronic states, simpler models combined with some empirical analysis can provide a more complete picture that complements computation. The active electron approximation provides an initial basis for the discussion of diradical species. This model considers the electronic states obtained from interaction of two electrons occupying two molecular orbitals and has been discussed and developed by various authors. ${ }^{29-32}$ Though this model is far from perfect, it typically provides a qualitatively correct picture and a useful framework to link properties such as oxidation potentials. The model results in four possible states; a triplet and three singlets. Of the latter, one involves the two spin- paired electrons occupying separate orbitals, (the open-shell singlet) and the remaining two involving two electrons occupying one orbital leaving the other orbital empty (the CT singlets). The energy difference between these states depends upon the Coulombic repulsion between the electrons, the exchange interaction between the electrons, the energy difference between the orbitals, and the "resonance" or "hopping" integral between the orbitals. We use the notation and description of Michl et al., ${ }^{14}$ who describe energies of these states in terms of the exchange integral $K$, a Coulombic term $K^{\prime}$ (approximately half the onsite repulsion energy, $U$ of the Hubbard model), the energy difference between the orbitals $\delta$, and an interaction term $\gamma$, corresponding to approximately twice the resonance or (hopping or transfer) integral. Furthermore, orbitals are chosen such that $\delta \geq 0$. In the case of the species under discussion, logical choices of orbitals are the verdazyl SOMO, and the highest occupied orbital of the substituted aryl fragment. These are depicted in Figure 7 below.
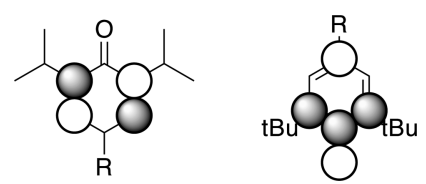

Figure 7. Representation of the verdazyl and phenoxyl SOMOs showing the differing symmetry.

Because of the differing symmetry of the two orbitals, (and assuming the molecule is close to planar) these two orbitals interact very weakly or not at all (i.e., $\gamma \approx 0$ ), though they may differ in energy. Under these circumstances the energies of the three singlet states of the system relative to the triplet are given by

$$
\begin{aligned}
& s_{\mathrm{os}}=2 K \\
& s_{\mathrm{CT} 1}=2 K^{\prime}+K-\sqrt{K^{2}+\delta^{2}} \\
& s_{\mathrm{CT} 2}=2 K^{\prime}+K+\sqrt{K^{2}+\delta^{2}}
\end{aligned}
$$

Assuming, for the moment, that the exchange interaction is small (for example if the two radicals were widely separated), these states can be approximated with the Lewis structures in Figure 8, and the energy of the CT states can be approximated using the redox potentials of the molecular components. That is:

$$
\begin{aligned}
& s_{\mathrm{CT} 1}=2 K^{\prime}-\delta=E\left(V^{+} / V^{\bullet}\right)-E\left(D^{\bullet} / D^{-}\right) \\
& s_{\mathrm{CT} 2}=2 K^{\prime}+\delta=E\left(V^{\bullet} / V^{-}\right)-E\left(D^{+} / D^{\bullet}\right)
\end{aligned}
$$

Though $\mathrm{K}^{\prime}$ would be expected to vary inversely with the number of atoms in the interacting fragments, ${ }^{33}$ the number of atoms contributing to the relevant $\pi$ orbitals in species 3,4 , and 


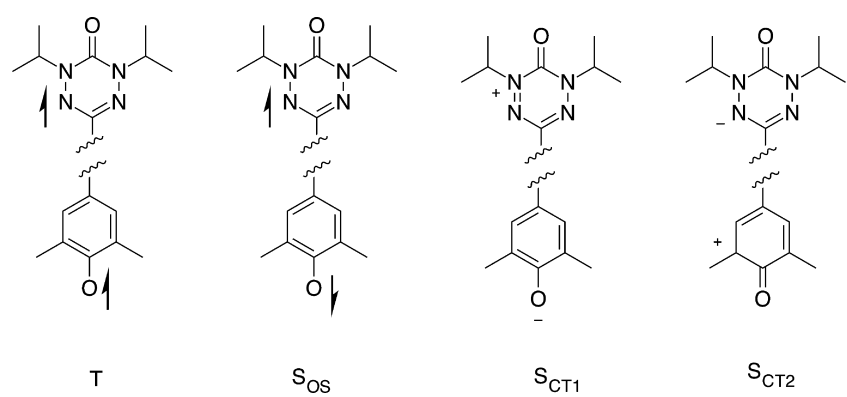

Figure 8. Nominal electronic configurations of the verdazyl-phenoxyl system.

7 does not change, and thus at this level, differences in energies of the various states can be largely attributed to differences in $\delta$, or possibly the exchange energy $K$.

Some relevant oxidation potentials are listed in Table 4.

This very simple model gives, for the phenol 3 , a ground CT state $0.89 \mathrm{eV}$ ( or $21 \mathrm{kcal} / \mathrm{mol}$ ) below the triplet state, while for the phenolate, the CT state is $0.77 \mathrm{eV}(18 \mathrm{kcal} / \mathrm{mol})$ above the triplet, which is now the ground state. The latter number compares favorably with the DFT calculations; thus, even this crude approximation is a useful starting point for understanding these systems.

Even before we consider how the exchange interaction might affect the $\mathrm{S}-\mathrm{T}$ energy splitting, we can obtain a better estimate of the CT state energy by considering how the redox potentials of the components are influenced by their mutual interaction. Rather than using potentials for the isolated verdazyl and phenolate listed in Table 1 we need to consider the more specific reactions shown in Scheme 4.

The potential $E_{1}$ can be obtained directly by measurement $(-0.15 \mathrm{~V}$ vs $\mathrm{SCE})$, but for $E_{2}$ some kind of estimation is required. It has already been noted that verdazyl oxidation potentials are weakly dependent on substituent effects at the 3 position. ${ }^{9}$ Using these published potentials and the Hammett constant for phenoxide ${ }^{39}$ gives an estimated potential of $\sim 0.5 \mathrm{~V}$ for $E_{2}$. Together, this gives the energy of the CT singlet as 16 $\mathrm{kcal} / \mathrm{mol}$ above the triplet.

These estimates are reasonable, but they completely ignore the exchange interaction $K$, which raises the energy of all three singlet states with respect to the triplet, lifting the degeneracy between the open-shell singlet and triplet. DFT calculations also place the open-shell singlet significantly above the triplet, which is consistent with our variable-temperature ESR experiment.

For the dimethylamino system, redox potentials (Table 4) predict the cation should have a closed-shell singlet ground state with a low-lying diradical excited state since the oxidation potential of the verdazyl is below that of the dimethylaniline. As with the phenolate 5 , we can further refine these estimates by using substituent constants to estimate the potentials for the reactions in Scheme 5 .

In this case, $E_{2}$ is directly determined by experiment $(+0.54 \mathrm{~V}$ vs SCE) and, as might be expected for a donor-substituted verdazyl, is slightly less than that for the phenyl verdazyl itself (Table 4). $E_{1}$, however, must be estimated. Other reports have shown that the oxidation potential of $N, N$-dimethylaniline is strongly dependent on substituent effects and correlates with the Hammett $\sigma$ parameter. ${ }^{36}$ Experiments on phenols indicate that a verdazyl is mildly electron-withdrawing when connected at the 3 position; ${ }^{9}$ consequently, we expect the oxidation potential of the dimethylaniline portion of molecule 4 to be higher than dimethylaniline itself. Using the previously determined Hammett parameter for a verdazyl we can estimate this potential to be ca. $+0.9 \mathrm{~V}$ versus SCE. Taken together, the interactions between the redox active groups thus work to stabilize the closed-shell CT species over the diradical. Using these potentials we can estimate the energy difference between diradical and CT states as $+0.35 \mathrm{~V}$ or $\sim 8 \mathrm{kcal} / \mathrm{mol}$. Again, the exchange interaction would be expected to raise the energy of the singlet state moving it closer to the excited triplet, but our experimental results suggest that this effect is not large enough to allow population of the triplet state at $77 \mathrm{~K}$.

Our results with dimethylamino verdazyl 4 contrast with Sakurai's studies on the corresponding nitronyl nitroxide cation, which is reported to be a ground-state triplet. ${ }^{8}$ This result is somewhat surprising, since the oxidation potentials for this system also predict a ground-state CT-type singlet. Substituent effects would also further stabilize the singlet-nitronyl nitroxide substituents were recently noted to increase the oxidation potential of $p$-phenylenediamines such that the nitronyl nitroxides were oxidized preferentially. ${ }^{40}$ One possible explanation for this anomaly is the exchange interaction, which has played only a small role in the discussion so far. As previously noted, introduction of an exchange parameter increases the energy of all three singlet states; thus, $S_{\mathrm{OS}}$ and $T$ are no longer degenerate, and there can be a clear triplet ground state. In cases where potentials favor the CT state $S_{\mathrm{CT} 1}$, if $\delta$ is sufficiently small, the exchange parameter may lift $S_{\mathrm{CT} 1}$ above the triplet state even though redox potentials suggest otherwise. This could explain the apparent triplet ground state observed in Sakurai's system; nitronyl nitroxides are harder to oxidize than verdazyls (by $\sim 0.1 \mathrm{~V}$ ), which may bring the potentials close enough that the triplet is favored. We note however that the triplet reported by Sakurai was only characterized by ESR as a small component of a mixture and that there may be other sources for this triplet signal.

DFT results on $7^{\prime}$ and $9^{\prime}$ in conjunction with the PCM also point to the importance of solvation in determining singlettriplet separation, especially when the singlet state in question is significantly polarized. Though indirectly related, the solvatochromism observed in the electronic spectrum of $\mathbf{5}$ and related phenolates, ${ }^{9}$ and the different absorption spectra of the polymorphs of 4 , both indicate the role of environment in electronic structure, and where the energy differences between states are small, such a role can potentially change the nature of the ground state. A notable example of this was reported recently with a doublet species; ${ }^{41}$ a similar effect with a triplet/ singlet system may well be achievable with a verdazyl or similar radical.

It is interesting to consider what external influences might trigger a switch from singlet to triplet ground state or vice versa. As long as the molecule has the same basic structure, $K^{\prime}$ will be largely unchanged, but changes in reduction potential of the radical components result in an effective change in $\delta$, while geometry changes can lead to changes in $K$ and $\gamma$. An example of the influence of geometry is provided by the $\mathrm{Cu}$ complexes of a pyridyl verdazyl that we have reported previously. ${ }^{42}$ In the case of the phenols, loss/gain of a proton provides sufficient change in $\delta$, but this is not possible for the amino species. A possible clue comes from the polymorphs of 4 . In these two systems, a $25 \mathrm{~nm}$ red shift of the lowest energy absorption is correlated with $\pi$-stacking of the dimethylaminophenyl groups. Since this absorption is associated with CT from the aromatic 
Table 4. Oxidation Potentials of Systems Related to Species 1-4

\section{Model system \\ oxidation potential vs SCE (/V)}<smiles>CC1=NN(C(C)C)C(=O)N(C(C)C)N1C</smiles>

$+0.6^{a}$

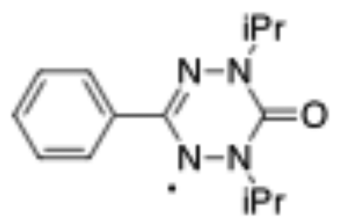

$+0.58^{b}$<smiles>CON1C(C)=[N+]([O-])C(C)(C)C1(C)C</smiles>

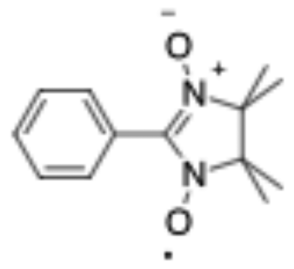

$+0.73^{c}$<smiles>CN(C)c1ccccc1</smiles><smiles>CC(C)(C)c1cccc(CBr)c1O</smiles><smiles>COc1c(C)cccc1Br</smiles>

$\mathrm{tBu}$

${ }^{a}$ From ref $34 .{ }^{b}$ From ref $11 .{ }^{c}$ From ref $35 .{ }^{d}$ From ref $36 .{ }^{e}$ From ref $37 .{ }^{f}$ From ref 38.

ring, the red shift indicates a decrease in oxidation potential of the donor of $\sim 0.1 \mathrm{~V}$, which may well be enough to favor a ground-state triplet over the singlet. It remains to find conditions where the verdazylium cation undergoes such $\pi$ stacking interactions to probe this possibility.

\section{CONCLUSION}

Phenoxide-substituted verdazyls are oxidized at the phenoxide to give triplet diradical species; however, the comparable dimethylaminophenyl verdazyl is oxidized to a diamagnetic verdazylium ion with a low-lying excited triplet. The dimethylaminophenyl verdazyl crystallizes in two polymorphs with different colors, suggesting that the electronic structure of these molecules can be significantly perturbed by environment. These results indicate that verdazyls are comparable and in some ways complementary to nitronyl-nitroxides in organic electronics and may suggest ways in which similar molecules may be incorporated into molecular switches, devices, and sensors.

\section{EXPERIMENTAL SECTION}

General. Cyclic voltammetry was performed in dry acetonitrile using $0.1 \mathrm{M}$ tetrabutylammonium perchlorate as supporting electrolyte and the ferrocene/ferricenium couple as an internal reference. 1,5Diisopropyl-3-(4'-dimethylaminophenyl)-6-oxoverdazyl (4) was syn- 


\section{Scheme 4}

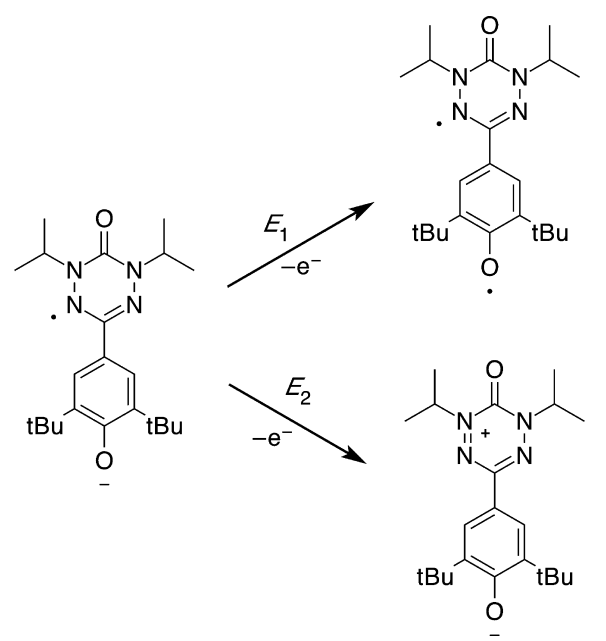

Scheme 5

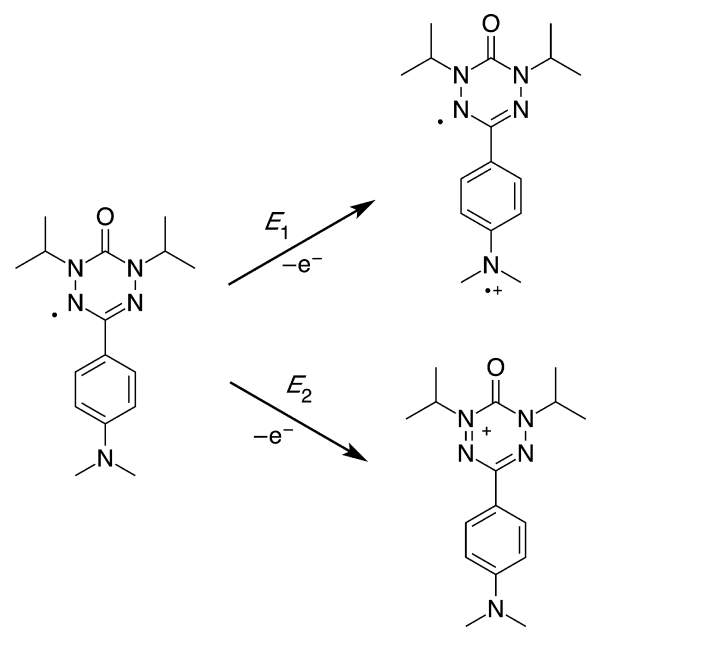

thesized as previously reported. ${ }^{9}$ Crystals of the purple $P 2_{1}$ form of this compound were grown by slow evaporation of a heptane solution. Spectrometric titrations were performed in acetonitrile solution, with the stepwise addition of a solution of the corresponding reagent $\left(\mathrm{Cu}(\mathrm{OTf})_{2}\right.$ or $\left.\mathrm{Et}_{3} \mathrm{~N}\right)$ in acetonitrile.

2,4-Diisopropyl-6-(3,5-di-tert-butyl-4-hydroxyphenyl)-1,2,4,5-tetrazinan-3-one (11). Sodium acetate $(0.932 \mathrm{~g}, 11.4 \mathrm{mmol})$ dissolved in minimal $\mathrm{H}_{2} \mathrm{O}$ was reacted with 3,5-di-tert-butyl-4-hydroxybenzaldehyde $(1.329 \mathrm{~g}, 5.7 \mathrm{mmol})$ and 2,4-diisopropylcarbonohydrazide bishydrochloride $^{10}(1.414 \mathrm{~g}, 5.7 \mathrm{mmol})$ dissolved in minimal EtOH, producing crude orange crystals. Recrystallization from methanol $(\sim 25$ $\mathrm{mL})$ gave the product $(0.370 \mathrm{~g})$. Evaporation of solvent gave a second crop $(0.132 \mathrm{~g})$. Product was rose-colored crystals $(0.502 \mathrm{~g}, 23 \%)$ with mp 221.1-236.6 ${ }^{\circ} \mathrm{C}$, IR $\nu_{\max }\left(\mathrm{NaCl}\right.$ plate), $\mathrm{cm}^{-1} 3467(\mathrm{NH} / \mathrm{OH})$, $2961(\mathrm{CH}), 1624(\mathrm{C}=\mathrm{O}) ;{ }^{1} \mathrm{H}$ NMR $\left(300 \mathrm{MHz}, \mathrm{CDCl}_{3}\right) 7.38$ (s, $2 \mathrm{H}), 5.37(\mathrm{~s}, \mathrm{OH}), 4.69($ septet, $2 \mathrm{H}, J=6.7), 4.52(\mathrm{t}, 1 \mathrm{H}, J=12.0), 3.74$ $(\mathrm{d}, 2 \mathrm{H}, J=12.0), 1.47(\mathrm{~s}, 18 \mathrm{H}), 1.17(\mathrm{~d}, 6 \mathrm{H}, J=6.7), 1.16(\mathrm{~d}, 6 \mathrm{H}, J=$ 6.7); ${ }^{13} \mathrm{C}$ NMR (75 MHz, $\mathrm{CDCl}_{3}$ ) 154.5, 154.3, 136.2, 126.4, 122.8, $104.4,71.6,47.8,34.4,30.2,19.6,18.4$; MS (EI, $70 \mathrm{eV}) \mathrm{m} / z$ (relative abundance) 390 (M+, 38), 275 (23), 234 (40), 233(27), 232 (100), 218 (34)

1,5-Diisopropyl-3-(3',5'-di-tert-butyl-4'-hydroxyphenyl)-6-oxoverdazyl (3). 2,4-Diisopropyl-6-(3,5-di-tert-butyl-4-hydroxyphenyl)$1,2,4,5$-tetrazinan-3-one $(11 ; 0.390 \mathrm{~g}, 1.00 \mathrm{mmol})$ was reacted with benzoquinone $(0.162 \mathrm{~g}, 1.50 \mathrm{mmol})$ in toluene as reported in the literature, ${ }^{10}$ with thin-layer chromatography on silica gel eluting with dichloromethane to monitor reaction progress. The product was recrystallized from methanol and chilled for $4.5 \mathrm{~h}$ to yield wine-red crystals $(0.319 \mathrm{~g}, 82 \%)$ with $\mathrm{mp} 193.7-196.2{ }^{\circ} \mathrm{C}$, IR $\nu_{\max }(\mathrm{NaCl}$ plate $)$, $\mathrm{cm}^{-1} 3435(\mathrm{NH}), 2963(\mathrm{CH}), 1659(\mathrm{C}=\mathrm{O}) ; \mathrm{MS}(\mathrm{EI}, 70 \mathrm{eV}) \mathrm{m} / \mathrm{z}$ (relative abundance) $387(\mathrm{M}+, 100), 345$ (22), 303 (84), 232 (38), 216 (28); ESR (toluene) $g=2.0066, a_{\mathrm{N} 1,5}=5.1 \mathrm{G}, a_{\mathrm{N} 2,4}=6.4 \mathrm{G}, a_{\mathrm{H}}=$ $1.8 \mathrm{G}$; UV-vis $\left(\mathrm{CH}_{3} \mathrm{CN}\right) \lambda, \mathrm{nm}\left(\varepsilon / \mathrm{L} \cdot \mathrm{mol}^{-1} \cdot \mathrm{cm}^{-1}\right)$ 418(990), $521(640)$.

Oxidation of 1,5-Diisopropyl-3-(3',5'-di-tert-butyl-4'-hydroxyphenyl)-6-oxoverdazyl. 1,5-Diisopropyl-3-( $3^{\prime}, 5^{\prime}$-di-tert-butyl-4' -hydroxyphenyl)-6-oxoverdazyl (2; $5 \mathrm{mg}, 0.013 \mathrm{mmol})$ was suspended in $1 \mathrm{~mL}$ of acetonitrile- $d_{3}$. Copper triflate $(4.7 \mathrm{mg}, 1$ equiv) was added, and the mixture was shaken vigorously as it turned from deep red to dark blue. The resulting solution of 1,5-diisopropyl-3-(3',5'-di-tertbutyl-4'-hydroxyphenyl)-6-oxoverdazylium cation (6) had ${ }^{1} \mathrm{H}$ NMR $\left(\mathrm{CD}_{3} \mathrm{CN}\right) 8.00(\mathrm{~s}, 2 \mathrm{H}), 6.32(\mathrm{~s}, 1 \mathrm{H}), 5.44$ (septet, $\left.2 \mathrm{H}\right), 1.67(\mathrm{~d}, 12 \mathrm{H})$, $1.51(\mathrm{~s}, 18 \mathrm{H})$. Subsequent to recording the NMR, triethylamine (one drop) was added to the solution. The solution immediately turned orange, and ${ }^{1} \mathrm{H}$ NMR revealed only signals due to triethylamine and solvent. A similar sample was prepared in a fused silica ESR tube, frozen using the cryostat, and ESR spectra recorded at temperatures from 4 to $100 \mathrm{~K}$.

Oxidation of 1,5-Diisopropyl-3-(4'-dimethylaminophenyl)-6-oxoverdazyl (4) with Copper Triflate. Oxidation of 1,5-diisopropyl-3(4'-dimethylaminophenyl)-6-oxoverdazyl was performed with copper triflate in a manner identical to that reported for radical 3. ${ }^{1} \mathrm{H}$ NMR of the resulting solution gave signals corresponding to 1,5-diisopropyl-3(4'-dimethylaminophenyl)-6-oxoverdazylium (9): NMR 8.30 (bs, $2 \mathrm{H}), 7.67$ (bs, 2H), 5.46 (septet, 2H), 3.26 (bs, 6H), $1.68(\mathrm{~d}, 12 \mathrm{H})$ along with additional unidentified peaks at 7.3 and $8.2 \mathrm{ppm}$.

Oxidation of 1,5-Diisopropyl-3-(4'-dimethylaminophenyl)-6-oxoverdazyl (4) with Silver Triflate. 1,5-Diisopropyl-3-(4'-dimethylaminophenyl)-6-oxoverdazyl $(6.8 \mathrm{mg}, 0.022 \mathrm{mmol}$ ) was dissolved in acetone $(1 \mathrm{~mL})$. To this solution was added $7 \mathrm{mg}(0.027 \mathrm{mmol})$ of silver triflate. The solution immediately turned yellowish-green. After $\sim 30$ min, the solution had changed to brown; after 90 min the solution was orange. The solution was filtered to remove a gray precipitate of silver and evaporated. Recrystallization from dichloromethane/ heptane gave $6.1 \mathrm{mg}(0.13 \mathrm{mmol}, 61 \%)$ of the triflate salt 9 as an orange solid. The UV-vis of this material was indistinguishable from an authentic sample of the ammonium cation generated by acidification of a solution of the radical. ${ }^{9}$ Crystals of this material were suitable for X-ray study. Full details of data collection and structure solution and refinement are available in the Supporting Information in .cif format.

Density Functional Theory Calculations. The geometries and energies of verdazyl radicals $7^{\prime}$ and $9^{\prime}$ in their triplet and singlet electronic states were computed using DFT with the B3LYP ${ }^{43,44}$ hybrid functional and $6-31 \lg (\mathrm{d}, \mathrm{p})$ basis set as implemented in Gaussian09. ${ }^{45}$ To test the effect of the polar solvent on the energy differences, the geometries were optimized in the presence of acetonitrile using the PCM. The BS formalism originally proposed by Noodleman ${ }^{12-14}$ for unrestricted Hartree-Fock (UHF) and later applied to DFT employs unrestricted wave functions for triplet $(\mathrm{Ms}=$ 1) and open-shell singlet $(\mathrm{Ms}=0)$ states. The molecular orbitals from a triplet ROHF/6-311g** calculation were used as input to the BSDFT singlet-state geometry optimization. The closed-shell singlet geometry was calculated with the restricted (R) DFT formalism. Broken symmetry wave functions have been used extensively in DFT calculations because of their computational efficiency and the B3LYP/ 6-311G** model in particular provides reliable singlet-to-triplet energy gaps. ${ }^{13,15-22}$ Geometry optimizations used an ultrafine grid of points for integration, and second derivatives were calculated at each step of the search. Default tolerances within Gaussian09 were otherwise used. All structures are minima on their respective potential energy surfaces.

\section{ASSOCIATED CONTENT}

\section{Supporting Information}

The Supporting Information is available free of charge on the ACS Publications website at DOI: 10.1021/acs.jpca.5b09295. 
Solid-state visible reflectance spectra for both forms of 4 . Magnetic data for both forms of $4 .{ }^{1} \mathrm{H}$ and ${ }^{13} \mathrm{C}$ NMR spectra for $11,{ }^{1} \mathrm{H}$ NMR spectra of solutions containing verdazylium ions 6 and 9. Curie plot for ESR of 7. Optimized DFT Cartesian coordinates, absolute electronic energies, and figures showing selected bond lengths of the different electronic states for $7^{\prime}$ and $\mathbf{9}^{\prime}$. (PDF)

Full details of the structure determination of the two polymorphs of $\mathbf{4}$ and the ammonium salt 10. (CIF) Crystallographic structure determination. (CIF) Crystallographic structure determination. (CIF)

\section{AUTHOR INFORMATION}

\section{Corresponding Authors}

*E-mail: david.brook@sjsu.edu. (D.B.)

*E-mail: ichimura@sfsu.edu. (A.I.)

\section{Notes}

The authors declare no competing financial interest.

\section{ACKNOWLEDGMENTS}

We thank the National Science Foundation (CHE-1058077 to D.J.R.B.) for financial support. We also thank A. Oliver for help with crystallography and J. Long and D. Freedman for help with magnetic data.

\section{REFERENCES}

(1) Kuhn, R.; Fischer-Schwarz, G. Von Zuckern abgeleitete freie Radikale. Monatsh. Chem. 1966, 97, 517-524.

(2) Kuhn, R.; Trischmann, H. Surprisingly Stable Nitrogenous Free Radicals. Angew. Chem., Int. Ed. Engl. 1963, 2, 155-155.

(3) Pütz, A.-M.; Schatzschneider, U.; Rentschler, E. Integrated Experimental and Computational Spectroscopy Study on the Protonation of the $\alpha$-Nitronyl Nitroxide Radical Unit. Phys. Chem. Chem. Phys. 2012, 14, 1649-1653.

(4) Chiang, J. C.; Macdiarmid, A. G. Polyaniline - Protonic Acid Doping of the Emeraldine Form to the Metallic Regime. Synth. Met. 1986, 13, 193-205.

(5) Wienk, M. M.; Janssen, R. A. J. Stable Triplet-State Di(Cation Radicals) of a Meta-Para Aniline Oligomer by "Acid Doping. J. Am. Chem. Soc. 1996, 118, 10626-10628.

(6) Sandberg, M. O.; Nagao, O.; Wu, Z. K.; Matsushita, M. N.; Sugawara, T. Generation of a Triplet Diradical from a Donor-Acceptor Cross Conjugate upon Acid-Induced Electron Transfer. Chem. Commun. 2008, 3738-3740.

(7) Ishiguro, K.; Ozaki, M.; Sekine, N.; Sawaki, Y. Acid-Base Equilibrium between Phenoxyl-Nitronyl Nitroxide Biradical and Closed-Shell Cation. A Magnetic pH Sensor. J. Am. Chem. Soc. 1997, 119, 3625-3626.

(8) Sakurai, H.; Izuoka, A.; Sugawara, T. Design, Preparation, and Electronic Structure of High-Spin Cation Diradicals Derived From Amine-Based Spin-Polarized Donors. J. Am. Chem. Soc. 2000, 122, 9723-9734.

(9) Chemistruck, V.; Chambers, D.; Brook, D. J. R. StructureProperty Relationships of Stable Free Radicals: Verdazyls with Electron-Rich Aryl Substituents. J. Org. Chem. 2009, 74, 1850-1857.

(10) Paré, E. C.; Brook, D. J. R.; Brieger, A.; Badik, M.; Schinke, M. Synthesis of 1,5-Diisopropyl Substituted 6-Oxoverdazyls. Org. Biomol. Chem. 2005, 3, 4258-4261.

(11) Gilroy, J. B.; McKinnon, S. D. J.; Koivisto, B. D.; Hicks, R. G. Electrochemical Studies of Verdazyl Radicals. Org. Lett. 2007, 9, 4837-4840.

(12) Noodleman, L. Valence Bond Description of Anti-Ferromagnetic Coupling in Transition-Metal Dimers. J. Chem. Phys. 1981, 74, 5737-5743.
(13) Noodleman, L.; Case, D. A. Density-Functional Theory of Spin Polarization and Spin Coupling in Iron-Sulfur Clusters. Advances in Inorganic Chemistry 1992, 38, 423-470.

(14) Noodleman, L.; Davidson, E. R. Ligand Spin Polarization and Antiferromagnetic Coupling in Transition-Metal Dimers. Chem. Phys. 1986, 109, 131-143.

(15) Yamaguchi, K.; Jensen, F.; Dorigo, A.; Houk, K. N. A Spin Correction Procedure for Unrestricted Hartree-Fock and M?llerPlesset Wavefunctions for Singlet Diradicals And Polyradicals. Chem. Phys. Lett. 1988, 149, 537-542.

(16) Yamaguchi, K.; Fueno, T.; Ueyama, N.; Nakamura, A.; Ozaki, M. Antiferromagnetic Spin Couplings Between Iron Ions in IronSulfur Clusters - A Localized Picture by the Spin Vector Model. Chem. Phys. Lett. 1989, 164, 210-216.

(17) Yamaguchi, K.; Tsunekawa, T.; Toyoda, Y.; Fueno, T. Abinitio Molecular-Orbital Calculations of Effective Exchange Integrals Between Transition-Metal Ions. Chem. Phys. Lett. 1988, 143, 371-376.

(18) Caballol, R.; Castell, O.; Illas, F.; de P. R. Moreira, I.; Malrieu, J. P. Remarks on the Proper Use of the Broken Symmetry Approach to Magnetic Coupling. J. Phys. Chem. A 1997, 101, 7860-7866.

(19) Ruiz, E.; Cano, J.; Alvarez, S.; Alemany, P. Broken Symmetry Approach to Calculation of Exchange Coupling Constants for Homobinuclear and Heterobinuclear Transition Metal Complexes. J. Comput. Chem. 1999, 20, 1391-1400.

(20) Shoji, M.; Koizumi, K.; Kitagawa, Y.; Kawakami, T.; Yamanaka, S.; Okumura, M.; Yamaguchi, K. A General Algorithm for Calculation of Heisenberg Exchange Integrals J in Multispin Systems. Chem. Phys. Lett. 2006, 432, 343-347.

(21) Lahti, P. M.; Ichimura, A. S.; Sanborn, J. A. Methodologies for Computational Studies of Quininoidal Diiminediyls: Biradical vs Dinitrene Behavior. J. Phys. Chem. A 2001, 105, 251-260.

(22) Ruiz, E.; Alvarez, S.; Rodriguez-Fortea, A.; Alemany, P.; Pouillon, Y.; Massobrio, C. Electronic Structure and Magnetic Behavior in Polynuclear Transition-Metal Complexes. In Magnetism: Molecules to Materials II, Molecule Based Materials; Wiley-VCH: Weinheim, Germany, 2001; pp 227-279.

(23) Tomasi, J.; Mennucci, B.; Cammi, R. Quantum Mechanical Continuum Solvation Models. Chem. Rev. 2005, 105, 2999-3093.

(24) Neese, F. Definition of Corresponding Orbitals and the Diradical Character in Broken Symmetry DFT Calculations on Spin Coupled Systems. J. Phys. Chem. Solids 2004, 65, 781-785.

(25) Malrieu, J. P.; Trinquier, G. A Recipe for Geometry Optimization of Diradicalar Singlet States from Broken-Symmetry Calculations. J. Phys. Chem. A 2012, 116, 8226-8237.

(26) Kitagawa, Y.; Saito, T.; Ito, M.; Shoji, M.; Koizumi, K.; Yamanaka, S.; Kawakami, T.; Okumura, M.; Yamaguchi, K. Approximately Spin-Projected Geometry Optimization Method and its Application to Di-Chromium Systems. Chem. Phys. Lett. 2007, 442, $445-450$.

(27) Ferre, N.; Guihery, N.; Malrieu, J. P. Spin Decontamination of Broken-Symmetry Density Functional Theory Calculations: Deeper Insight and New Formulations. Phys. Chem. Chem. Phys. 2015, 17, 14375-14382.

(28) Staab, H. A.; Gabel, G.; Krieger, C. Electron Donor-Acceptor Compounds, 44 Synthesis, Molecular Structure, and Electron-Donor Properties of Pseudogem-5,8,14,17-Tetrakis(Dimethylamino)[3.3]Paracyclophane. Chem. Ber. 1987, 120, 269-273.

(29) Salem, L.; Rowland, C. The Electronic Properties of Diradicals. Angew. Chem., Int. Ed. Engl. 1972, 11, 92-111.

(30) Bonacic-Koutecky, V.; Koutecky, J.; Michl, J. Neutral and Charged Biradicals, Zwitterions, Funnels in S1 and Proton Translocation: Their Role in Photochemistry, Photophysics and Vision. Angew. Chem., Int. Ed. Engl. 1987, 26, 170-189.

(31) Calzado, C. J.; Cabrero, J.; Malrieu, J. P.; Caballol, R. Analysis of the Magnetic Coupling in Binuclear Complexes. I. Physics of the Coupling. J. Chem. Phys. 2002, 116, 2728-2747.

(32) Calzado, C. J.; Cabrero, J.; Malrieu, J. P.; Caballol, R. Analysis of the Magnetic Coupling in Binuclear Complexes. II. Derivation of 
Valence Effective Hamiltonians from $\mathrm{Ab}$ Initio $\mathrm{CI}$ and $\mathrm{DFT}$ Calculations. J. Chem. Phys. 2002, 116, 3985-4000.

(33) Verot, M.; Rota, J.-B.; Kepenekian, M.; Guennic, B. L.; Robert, V. Magnetic and conduction properties in $1 \mathrm{D}$ organic radical materials: an ab initio inspection for a challenging quest. Phys. Chem. Chem. Phys. 2011, 13, 6657-6661.

(34) Brook, D. J. R.; Yee, G. T. Radical-Radical Interaction Through a Saturated Link: Methylenebis-6-Oxoverdazyl. J. Org. Chem. 2006, 71, 4889-4895.

(35) Budnikova, Y.; Gryaznova, T.; Kadirov, M.; Tret'yakov, E.; Kholin, K.; Ovcharenko, V.; Sagdeev, R.; Sinyashin, O. Electrochemistry of Nitronyl and Imino Nitroxides. Russ. J. Phys. Chem. A 2009, 83, 1976-1980.

(36) Parker, V. D.; Tilset, M. Facile Proton-Transfer Reactions of N,N-Dimethylaniline Cation Radicals. J. Am. Chem. Soc. 1991, 113, $8778-8781$.

(37) Kanamori, D.; Furukawa, A.; Okamura, T.; Yamamoto, H.; Ueyama, N. Contribution of the Intramolecular Hydrogen Bond to the Shift of the pK(a) Value and the Oxidation Potential of Phenols and Phenolate Anions. Org. Biomol. Chem. 2005, 3, 1453-1459.

(38) Hapiot, P.; Pinson, J.; Yousfi, N. Substituent Effects on The Redox Properties of Phenolates in Acetonitrile. One Electron Redox Potentials. New J. Chem. 1992, 16, 877-881.

(39) Exner, O. In Correlation Analysis in Chemistry: Recent Advances; Chapman, N. B., Shorter, J., Eds.; Plenum: New York, 1978; p 439.

(40) Ito, A.; Kurata, R.; Sakamaki, D.; Yano, S.; Kono, Y.; Nakano, Y.; Furukawa, K.; Kato, T.; Tanaka, K. Redox Modulation of paraPhenylenediamine by Substituted Nitronyl Nitroxide Groups and Their Spin States. J. Phys. Chem. A 2013, 117, 12858-12867.

(41) Nishida, S.; Morita, Y.; Fukui, K.; Sato, K.; Shiomi, D.; Takui, T.; Nakasuji, K. Spin Transfer and Solvato-/Thermochromism Induced by Intramolecular Electron Transfer in a Purely Organic Open-Shell System. Angew. Chem., Int. Ed. 2005, 44, 7277-7280.

(42) Brook, D. J. R.; Yee, G. T.; Hundley, M.; Rogow, D.; Wong, J.; Van-Tu, K. Geometric Control of Ground State Multiplicity in a Copper(I) Bis(verdazyl) Complex. Inorg. Chem. 2010, 49, 8573-8577. (43) Becke, A. D. Density-Functional Exchange-Energy Approximation with Correct Asymptotic-Behavior. Phys. Rev. A: At., Mol., Opt. Phys. 1988, 38, 3098-3100.

(44) Lee, C. T.; Yang, W. T.; Parr, R. G. Development of the ColleSalvetti Correlation-Energy Formula into a Functional of the ElectronDensity. Phys. Rev. B: Condens. Matter Mater. Phys. 1988, 37, 785-789.

(45) Frisch, M. J.; Trucks, G. W.; Schlegel, H. B.; Scuseria, G. E.; Robb, M. A.; Cheeseman, J. R.; Scalmani, G.; Barone, V.; Mennucci, B.; Petersson, G. A., et al. Gaussian 09; Gaussian, Inc: Wallingford, CT, 2009. 